\title{
From Laser Pulse Propulsion to Fusion Pulse Propulsion: An Evolutionary Approach
}

\author{
Brice Cassenti ${ }^{1}$ \\ University of Connecticut, Storrs, CT, 06269 \\ Robert Budica ${ }^{2}$ \\ Technology Consultant, Laguna Hills, California 92653 \\ Les Johnson ${ }^{3}$ \\ NASA Marshall Space Flight Center, Huntsville, AL, 35801 \\ and \\ Terry Kammash ${ }^{4}$ \\ University of Michigan, Ann Arbor, MI 48109
}

\begin{abstract}
Laser pulse propulsion is a technology that can deliver attractive performance as measured not only by specific impulse, but also by thrust and power-to-weight ratios for voyages throughout the solar system. The simplest laser pulse propulsion operates by vaporizing coated plastic pellets in a thrustgenerating nozzle assembly. Initial estimates show this should not only be competitive with current advanced propulsion systems but more advanced laser propulsion versions using pellets that are filled with a deuterium-tritium mixture can significantly increase the exhaust energy). These pellets have the potential to move well beyond the ultimate performance of currently proposed advanced propulsion systems. Advanced systems could use U-238 coated propellant pellets filled with D-T to extend the energy balance beyond breakeven. Excess neutrons captured in a U-238-containing nozzle assembly will further increase the performance. Later improvements could replace the laser with electron beams, ion beams, or even antiproton beams. All of the improvements depend on the feasibility of developing a competitive laser-heated pulse pellet system. Once this system is developed, improvements can be added incrementally, leading to game-changing pulsed-nuclear fusion propulsion that would redefine the future of space exploration.
\end{abstract}

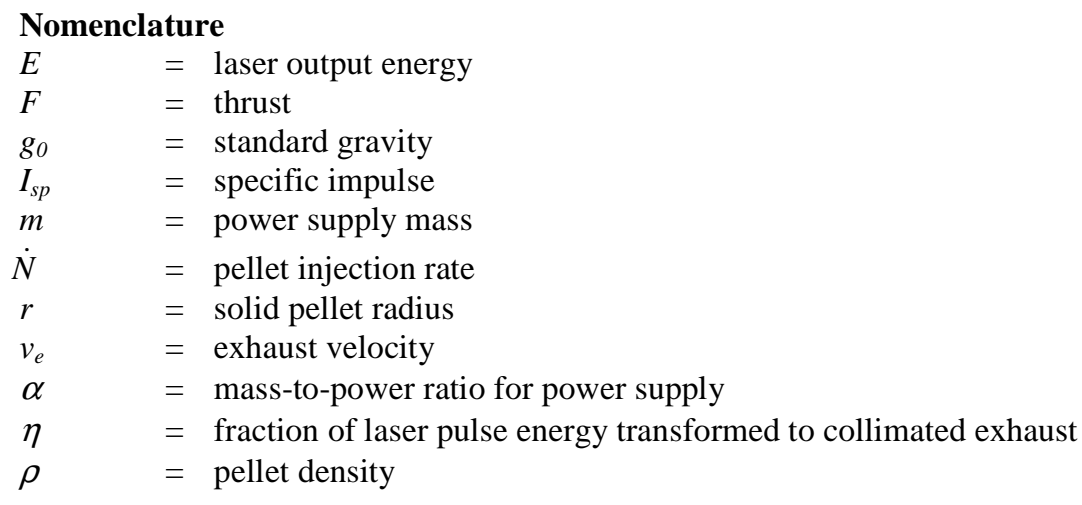

${ }^{1}$ Professor in Residence, Mechanical Engineering, University of Connecticut, 191 Auditorium Rd. U-3139, Storrs, CT 06269, AIAA Associate Fellow.

${ }^{2}$ Consultant, Laguna Hills, CA

${ }^{3}$ Technical Assistant, Advanced Concepts Office, NASA Marshall Spaceflight Center, Alabama, AIAA Member

${ }^{4}$ Professor Emeritus, University of Michigan, Ann Arbor, MI AIAA Associate Fellow

1

American Institute of Aeronautics and Astronautics 


\section{Introduction}

T asers have been considered as a means to propel aerospace vehicles for over 50 years. Concepts include: 1) Lpropulsion by direct photon momentum transfer ${ }^{1,2}$ and 2) laser-heated propellents ${ }^{3,4}$. Proposals for the use of lasers in propulsion are frequently considered for driving inertial confinement fusion ${ }^{5}$. The difference between laserheated propellants and laser-driven inertial confinement fusion is the requirement for considerably more power. This connection can be exploited to create high specific impulse, high thrust space propulsion.

The earliest concepts in laser propulsion used the momentum of the emitted photons as thrust. For example, Eugene Sanger ${ }^{1}$ considered the annihilation of electrons and positrons into gamma rays. Later, Robert Forward ${ }^{2}$ considered lasers focused by lenses the size of the Moon to focus lasers in orbit about the Sun. Obviously both of these are well beyond any current technological projections.

A concept that could be pursued today is laser-heated propellents ${ }^{4,6}$. The exhaust velocity is not limited by the chemical energy stored in the fuel and oxidizer. These propellants could theoretically produce exhaust velocities in excess of $10 \mathrm{~km} / \mathrm{s}$, or thrusts large enough to lift payloads from the surface of the earth ${ }^{6}$. Instead of heating the propellant directly, pellets could be vaporized, and the resulting vapor could be used to provide thrust. The ultimate goal in laser-heated propellants is fusion propulsion. Fusion would not only provide the necessary propulsion for deep space lunar and interplanetary missions, but would also propel interstellar precursor missions and even robotic missions to the nearer stars 7,8 .

Laser pulse propulsion is a technology that can deliver exceptional performance measured not only by specific impulse, but also by thrust and power-to-weight ratios for voyages throughout the solar system. The simplest laser pulse propulsion operates by vaporizing coated plastic pellets in a thrust-generating nozzle assembly as shown in Figure 1. Initial estimates show a fully developed laser-heated pellet pulse propulsion system should not only be competitive with current advanced propulsion systems, but with more advanced laser propulsion versions using pellets that are filled with a deuterium-tritium (D-T) mixture (to increase the exhaust energy). These pellets have the potential to move well beyond the ultimate performance of currently proposed advanced propulsion systems. Advanced systems could use U-238 coated propellant pellets filled with D-T to extend the energy balance beyond break-even. Excess neutrons captured in a U-238-containing nozzle assembly will further increase the performance. All of these improvements depend on the feasibility of developing a competitive laser-heated pulse pellet system. Once this is developed, improvements can be added incrementally, leading to a game-changing pulsed-nuclear fusion propulsion that would redefine the future of space exploration. Later improvements could replace the laser with ion beams or even the beams of antiprotons, with the ultimate goal being the development of a working pulsenuclear fusion propulsion system.

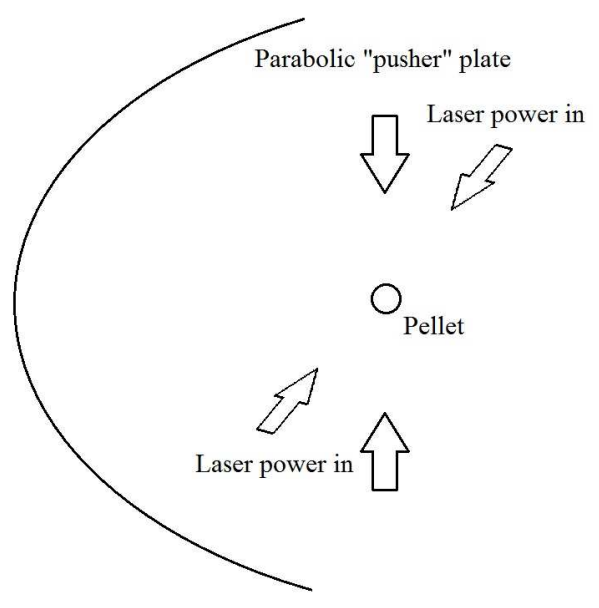

Figure 1. Pellet with parabolic pusher plate

The primary problem in fusion propulsion is reducing the technical risk in the development of the pulse-fusion propulsion systems. This problem is best approached by developing pulse propulsion systems that do not need to ignite a fusion reaction but are still competitive with other technologies. A possible approach would be to use lasers or particle beams to heat pellets and use the thermal energy for propulsion. Such a system would drive the 
development of robust, lightweight power systems, efficient lasers, and possibly even compact particle beam accelerators. Later fusion fuel could be added to the pellets to boost the specific impulse with little to no loss in thrust and specific impulse. A self-sustaining fusion reaction would not be required during the development since power would be provided externally. Only the highest specific impulse (on the order of 1,000,000 seconds) would actually make use of the fusion breakeven technology.

\section{Laser-Heated Pulse Pellet Propulsion}

Chemical rockets have been the mainstay of deep space propulsion for the last 50 years. Their high thrust/weight ratio and $I_{s p}$ performance can readily lift payloads from the surface of the earth to orbit, but for nonHohmann trajectory deep space missions, the propellant mass required becomes excessive. There are options for propulsion systems that could potentially alleviate the high propellant masses that chemical propulsion requires. Unfortunately, as the specific impulse increases, the thrust generally decreases. The result being no reduction in mission time, but there is a significant decrease in propellant mass. Hence, current technologies do not seem to offer relief from the long mission times required for missions throughout the solar system.

In Table 1, chemical propulsion is compared to three other technologies: 1) nuclear thermal propulsion which was demonstrated in the NERVA program in the early 1970s, 2) solar-heated propellants which are yet to be conclusively demonstrated, and 3) electric propulsion which is already in limited use. Electric propulsion consists of several types, including: 1) Hall Effect thrusters with a specific impulse (typically 2000-2500 seconds), 2) MHD/MPD thrusters (which includes VASIMR ${ }^{9}$ ) with a specific impulse (typically 1200-5000 seconds), 3) arc jets with a specific impulse (typically 900-1200 seconds), and 4) ion, or electrostatic thrusters (with specific impulse in the 1200-2500 second range). As seen in Table 1, the thrust-to-weight ratio for the highest specific impulse rockets is quite low. Pulse-nuclear fusion propulsion technologies can circumvent the problem of low thrust at high specific impulse with a specific impulse of over 200,000 seconds and a thrust-to-weight ratio of $0.1^{10}$, but would potentially require a long, risky development program before they could be demonstrated.

Table 1. Typical Performance of Propulsion Systems ${ }^{11}$

\begin{tabular}{lcc}
\hline Propulsion System & Specific Impulse - seconds & Thrust-to-Weight \\
\hline \hline Chemical & $200-600$ & $5 \times 10^{-3}-200$ \\
Nuclear Thermal & $750-1000$ & $5 \times 10^{-2}-50$ \\
Solar-Heated Hydrogen & $600-1000$ & $5 \times 10^{-4}-5 \times 10^{-2}$ \\
Ion & $1000-3500$ & $1 \times 10^{-6}-8 \times 10^{-5}$ \\
\hline
\end{tabular}

The primary problem is reducing the technical risk in the development of pulse fusion propulsion systems. The problem is best approached by gradually stepping up to pulse fusion propulsion by developing pulse propulsion systems that do not need to ignite a fusion reaction but are still competitive with the technologies in Table 1. A possible approach would be to use lasers or particle beams (e.g., the Daedalus project ${ }^{7}$ ) to heat pellets and use the resultant thermal energy for propulsion. Free electron lasers would be particularly well-suited since they can be tuned to a desirable frequency, and would bridge the gap from conventional laser heating to particle beam heating. Such a system would drive the development of robust, lightweight power systems, efficient lasers, and possibly even compact particle beam accelerators. Note that although the lasers and the particle beam accelerators would have a very high specific impulse, they would also provide exceedingly low thrust and thrust/power ratios. Heating pellets instead of directly using the lasers would increase the thrust at the expense of specific impulse. Fusion fuel could then be added to the pellets to boost the specific impulse with little to no loss in thrust. A self-sustaining fusion reaction would not be required during the development of a pulse fusion propulsion system since power could be provided externally. Only the highest specific impulse (on the order of 1,000,000 seconds) would actually make use of the more challenging breakeven fusion technology.

The question that remains is whether a laser-heated pulse pellet system can be competitive with other space propulsion systems. A rough model can be developed to estimate the performance by borrowing the analyses from the inertial confinement fusion community ${ }^{12}$. If a fraction, $\eta$, of the laser pulse energy, $E$, becomes collimated 
exhaust using a suitably shaped reflector/nozzle by heating a pellet of radius, $r$, and density, $\rho$, then the specific

impulse, $I_{s p}$, is approximately given by

$$
I_{s p}=\frac{v_{e}}{g_{0}}=\frac{1}{g_{0}} \sqrt{\frac{3 \eta E}{2 \pi \rho r^{3}}},
$$

where $v_{e}$ is the exhaust velocity and $g_{0}$ is the acceleration of gravity at the surface of the earth.

In equation (1) the work flow of the nozzle has been neglected, effectively replacing the specific heat at constant pressure with the specific heat at constant volume. Note that the efficiency, $\eta$, can be taken as the overall efficiency of the laser system.

The thrust-to-mass ratio can be found by taking the thrust, $F$, as the mass flow rate multiplied by the exhaust velocity. Since the mass flow rate is just the pellet mass times the pellet injection rate, $\dot{N}$, the thrust can be written as

$$
F=\frac{4}{3} \pi \rho r^{3} \dot{N} I_{s p} g_{0}
$$

The mass of the laser pulse propulsion system will be assumed to consist mainly of the power supply for the laser. Assuming a fission reactor ${ }^{9}$ is used then the mass, $m$, of the power supply will conservatively require 1-10 $\mathrm{kg} / \mathrm{kWe}^{9,13}$ of the laser power. In reference 13 , a value of $3.2 \mathrm{~kg} / \mathrm{kWe}$ was used. The thrust-to-weight ratio (i.e., the thrust-to-mass of the power supply in units of the earth's gravity) becomes,

$$
\frac{F}{m g_{0}}=\frac{4 \pi \rho r^{3} \dot{N} I_{s p}}{3 \alpha E}
$$

where $\alpha$ is the mass-to-power ratio for the power generation. Equations (1) and (3) can be combined to yield a relationship between the thrust-to-weight ratio for the power supply and the specific impulse in seconds. The result is

$$
\frac{F}{m g_{0}} I_{s p}=\frac{2 \eta}{\alpha g_{0}^{2}}
$$

Estimates for the performance can now be completed. Consider pellets with an overall density of $1 \mathrm{~g} / \mathrm{cm}^{3}$ and a diameter of $2 \mathrm{~mm}$. If the conversion efficiency was 10\%, then a $1 \mathrm{MJ}$ laser pulse would have a specific impulse of about 22,000 seconds. For 100 pellets per second (i.e., a total power of $100 \mathrm{MW}$ ), the thrust would be $90 \mathrm{~N}$, and the thrust-to-weight would be about $3 \times 10^{-5}$. Although certainly very advanced, this would outperform any of the systems in Table 1 in specific impulse and equal the performance in thrust-to-mass for ion propulsion systems. Note that an increase in the pellet mass will yield additional thrust at the expense of specific impulse making laser-heated pulse propulsion competitive with the other technologies listed in Table 1. The efficiency of the laser system, including the laser itself and any beam-shaping reflector system, is not high enough to warrant an estimate of $10 \%$. However, even $1 \%$ would only decrease the specific impulse by a factor of 3, thrust and thrust-to-weight by a factor of 10, and laser-heated pellets would remain competitive. If electron beams or particle beams were used, the efficiency would be better but the mass would increase. A conversion efficiency of $1 \%$ is near the minimum, while, of course, an efficiency of $100 \%$ is the maximum. Hence, we can provide bounds on laser-heated pulse pellet propulsion and add them to Table 1 . The specific impulse would vary from about 7,000s-70,000 seconds, and the thrust-to-weight from $10^{-5}$ to $10^{-4}$. This is certainly competitive, and warrants serious consideration for deep space propulsion.

Laser-heated pulse pellet propulsion systems are well-suited to variable-specific impulse rockets. Ideally, the exhaust velocity should be equal to the total change in speed of the rocket. The rocket would then convert all of the energy released in the propellant into the kinetic energy of the motion.

In laser-heated pellets, variable specific impulse can be achieved via three methods: 1) changing the pellet radius, 2) changing the material mix to produce different effective densities, and 3) changing laser-power-ratio to pellet-weight-ratio. The second choice is better than the first; it would minimally affect the laser system if the radius were kept constant. The third choice changes the effective pellet vapor temperature, and changes exhaust velocity and specific impulse as a result. 
Of course, a change in specific impulse will usually produce a change in thrust. Missions would have high thrust initially, where low specific impulse propellants would not require excessive speed changes (e.g., to escape from low-Earth orbit), and would result in a decrease in the mission time (i.e., there is no need to spiral out of orbit). This is the same capability as proposed in VASIMIR ${ }^{9}$.

\section{Pellet Improvements}

Although laser-heated pulse pellet propulsion may be competitive today with existing proposals, it is the promise for the future that makes it an excellent choice for development. Using the Laser Accelerated Plasma Propulsion System (LAPPS) proposed by Terry Kammash ${ }^{13}$, the exhaust velocity could be significantly improved. Adding propellant or explosives to the pellet ${ }^{14,15}$ would further improve the thrust, but decrease the specific impulse. The performance can also be improved if a hollow pellet is filled with deuterium and tritium (D-T); the intense laserinduced compression heating will produce some fusion reactions and a significant additional energy release beyond the laser input. Of course, breakeven is not required. However, any additional fusion energy would increase the performance at no cost to a pellet consisting of D-T without fusion. A shell of uranium 238 would provide a sink and a source for neutrons by adding fission energy to the pellet. The shell would not only provide inertial confinement for the internal D-T fuel, it would also add energy by absorbing fast neutrons emitted by the fusion of $\mathrm{D}-\mathrm{T}$ in the pellet's hollow core once the uranium is compressed. Uranium fission would also recompress any leftover/unreacted D-T, resulting in the creation of additional fusion reactions.

Additional improvements to the pellet include adding lithium ${ }^{23}$ and igniting the pellet from the inside out (i.e., Magnetically Insulated Inertial Confined Fusion or $\mathrm{MICF}^{22}$ ). . In MICF, the laser beam passes through a hole to the pellet center where it ignites a fusion reaction that propagates toward the outside. MICF will also significantly increase transient magnetic fields, further confining the plasma. The outside of the pellet has a high-density shell that inertially confines the fusion reaction for the time it takes the compression wave to travel through the pellet and shell.

In pellets consisting of lithium and deuterium, the absorption of a neutron by lithium- 6 will fission the lithium. The neutron absorption will create the helium and tritium required for fusion. This would allow for the construction of non-cryogenic pellets, but would require a significant increase in the density, causing a considerable increase in the power required for the compression.

\section{Driver Improvements}

Improvements to the laser driver are just as important for achieving nuclear pulse propulsion as improvements to the laser itself. Possible improvements include: 1) the use of a free electron laser (FEL) to produce intense collimated beams of X-rays, 2) replacing the laser with electron or ion beams, ${ }^{7,18}$, and 3) the use of antiprotons as a trigger for fission or fusion reactions ${ }^{22,25}$.

FELs can theoretically be tuned to any frequency, but their bulk and weight require considerable power. Nevertheless, it should be possible to produce an intense X-ray beam that could efficiently and effectively compress pellets. This is especially important if lithium- 6 and deuterium are used as the fusion fuel.

An obvious improvement over lasers is to use electron or ion beams. Since the beams now consist of particles with mass, they carry significantly more momentum and are more effective than photons at inertial confinement. The Daedalus Project ${ }^{7}$, which considered an interstellar fly-by mission, came to the conclusion that inertial confinement fusion using ion beams for the compression of the pellet was the most effective means by which to produce the high specific impulse (about 1,000,000 seconds) required.

The highest energy densities can be obtained by storing the energy as antimatter, preferably in the form of antiprotons. Of course, producing and storing antiprotons in large quantities is not possible today, but particle accelerators that use antiproton beams have been built. Today, the quantities that can be produced annually are small (about 10 nanograms per year ${ }^{25}$ ), but even small quantities would be useful as a trigger for fission ${ }^{20}$ or fusion reactions. For example, in reference 22, antiprotons are annihilated in uranium at the center of an MICF pellet. The uranium fissions and the high energy nuclear fragments are then used to begin a fusion reaction that propagates through the pellet. Recall that MICF produces intense magnetic fields that should contain the expansion of the fusion fuel ions long enough to allow the fusion reaction to begin. It should also be noted that if the antiprotons have the correct energy, it should be possible to precisely annihilate them on the uranium at the center of the pellet without the need for a hole ${ }^{21}$. 


\section{System Considerations}

Of course, many major technical challenges remain. For example, the efficiency (light-energy-out-to-energyin ratio) of lasers is generally low (some efficiency levels are as low as a few percent), and the choice of the laser system must match the properties of the pellets ${ }^{22}$ so that the rate of absorption is nearly $100 \%$. Note that the process starts with absorption heating of the pellet surface producing plasma that absorbs more light and transfers heat to the solid surface below, vaporizing it and continuing the process. The heated plasma expands and compresses the pellet surface below, creating an inward-radial acceleration/implosion. Specialized coatings would help. A pellet heating repetition rate of 100 laser shots per second is quite high. The fast refresh rate for high-power lasers will be a challenge. The power system must be able to recharge the laser in the short time available. The pellets must be targeted accurately ${ }^{23}$; this task may require an elaborate control system. For example, control system difficulties can be reduced if the pellets are part of a pearl-like necklace fed into the laser thruster's focal point. All of these engineering problems are being addressed by the inertial fusion community, along with many other problems shared by these two technologies. As a result, advancements in laser-heated pulse propulsion technology or inertial confinement fusion technology can be applied to the other.

Lightweight power systems must also be developed, but are currently being addressed by the space community. Addressing these aspects would help to decrease the system mass. Nuclear fission power can provide values that are even lower than the $3.2 \mathrm{~kg} / \mathrm{kW}$ assumed and might actually decrease to values less than one ${ }^{9}$.

The engine nozzle is an important consideration ${ }^{24}$. It appears that a transpiration-cooled nozzle structure might be desirable so that the coolant becomes part of the thrust-producing exhaust. If the nozzle is much larger than the pellet, the nozzle surface may see rarefied flow (i.e., the mean free path of gas molecules or atoms is much larger than physical dimensions of elements with which they interact). This will drive the nozzle geometry to be parabolic. If the nozzle is smaller, and its surface sees continuum flow, then hypersonic flow will drive the shape to be more conventional as in bell rocket nozzles.

For lower specific impulse systems, where the temperatures are relatively low, a simple parabola of revolution "pusher plate" nozzle may be adequate. If the pusher plate is cooled, then the energy of the heated coolant could be fed back into the lasers to increase the overall efficiency. Finally, in the future, an effective magnetic nozzle can be developed for the case of very high specific impulse laser-heated pellets where contact with the flow would rapidly deteriorate the nozzle wall. A magnetic nozzle may be the optimal solution in this case.

In all cases, it may be necessary to keep the size and release of charged particles very low so as not to pose a radiation threat that adds particles to the Van Allen belts.

We need to clearly state the difference between the fusion power challenge, which must exceed breakeven (i.e., energy-out-to-energy-in ratios greater than one) compared to pulse propulsion applications which do not need to achieve breakeven to be practical. Laser-heated pulse pellet propulsion focuses on simply vaporizing pellets - as the "working fluid" - by using lasers with the potential to evolve into pulse fusion propulsion systems. Recall that this technology is designed to evolve from a simple laser-vaporized pellet pulsed propulsion technology to the concept where additional energy is extracted from D-T fusion. It does not go beyond breakeven, but significantly increases the performance (both specific impulse and power-to-mass). In the next evolutionary step, fast neutrons released from the D-T fusion reaction can be used to fission U-238 nuclei. A layer of U-238 will be used to coat the pellets. This coating will further increase the specific impulse and power-to-mass ratio of the system. Depending on pellet size and ratio of D-T to U-238, the energy produced could even exceed breakeven. Exceeding breakeven will occur when the U-238 undergoing fission recompresses the unreacted D-T "residue" that will then be fusing the D-T without increasing the power used by the lasers. In the final system, to save mass, lasers may be replaced with light ion beams or even antimatter to trigger the fusion reaction ${ }^{17}$.

The infrastructure for most of the technology is currently present within the United States. The laser fusion community is actively conducting further research at the National Ignition Facility ${ }^{25}$. The knowledge gathered at the facility is a key component of the LHPPP (Laser-Heated Pellet Pulse Propulsion) development program. The fusion community power activities will also be a critical contribution to the future of LHPPP ${ }^{25}$. Not only is laser heating an important component of LHPPP but ion propulsion will also provide contributions vital to LHPPP. The temperatures present in the exhaust products will be high enough to use routine ion propulsion techniques.

\section{Development and Application Missions}

Initial LHPPP systems will consist of ground and flight demonstrations followed by an initial operational system. Table 2 below contains mass estimates for each of these demonstrations and systems. Based on a $\alpha$ of 3.0 $\mathrm{kg} / \mathrm{kWe}$, the power supply of the initial operational system in Table 2 would provide $12 \mathrm{kWe}$. Assuming a pellet density $(\rho)$ of $1.0 \mathrm{~g} / \mathrm{cm}^{3}$, a pellet radius $(r)$ of $0.3 \mathrm{~mm}$, a pellet injection rate $(\dot{N})$ of 1000 per second, and an energy 
transfer efficiency $(\eta)$ of 01 , the operational system would have an $I_{s p}$ of $470 \mathrm{~s}$ and an acceleration of $0.17 \mu \mathrm{G}$. This clearly is not a competitive value for the power. Nevertheless, it could provide attitude control and orbital corrections for geosynchronous satellites.

Equation (4) can be used to examine the trade-off between specific impulse and thrust-to-power supply weight for the case of an $\alpha$ of $3.0 \mathrm{~kg} / \mathrm{kWe}$ and an energy transfer efficiency, $\eta$, (energy from the power supply to the pellet exhaust velocity) of 01 . For the case in Figure 2 the parameter, $2 \eta /\left(\alpha g_{0}^{2}\right)$, is 0.69 , and represents the performance for the operational mission in Table 1. Clearly either the efficiency for the energy transferred to the pellet exhaust must be increased and/or the power supply mass must be decreased relative to the energy output. Nuclear power is clearly a feasible solution.

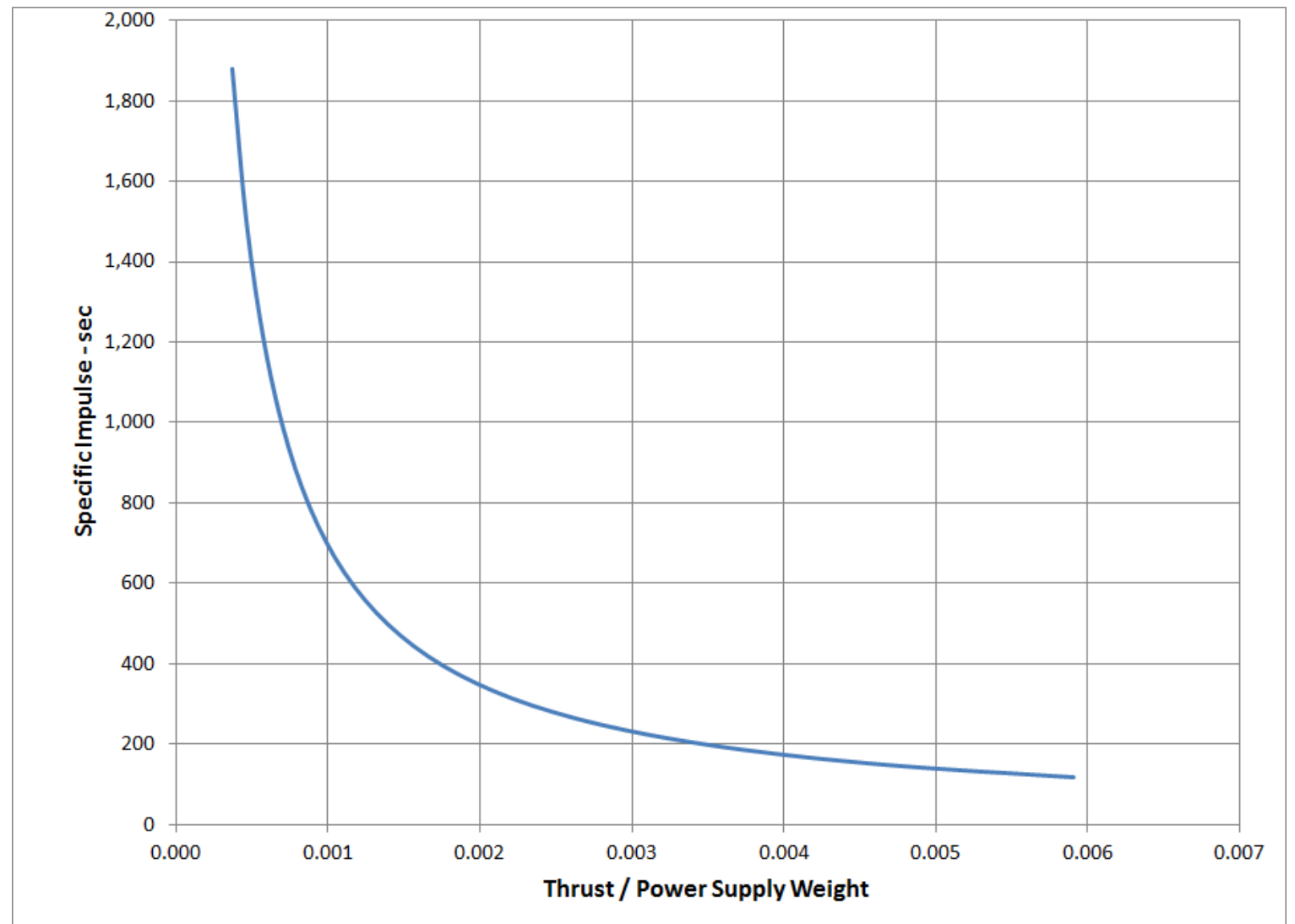

Figure 2. Pellet Thrust-to-weight and specific impulse for the operational mission in Table $1(\eta=0.1, \alpha=3)$.

The promise is in larger units powered by nuclear reactors. For example, a 1-year piloted Mars mission becomes a reality for a specific impulse of 20,000 seconds and a thrust-to-weight ratio of about $0.05^{27}$. The mission might be accomplished using pellets enhanced by fusion/fission reactions without the need to achieve breakeven with respect to the laser input energy. The evolution to nuclear pulse fusion propulsion with self-sustained fusion reactions, though not required for our laser propulsion concept, will allow the performance to reach more than 200,000 seconds and accelerations of 0.07 times Earth's gravity ${ }^{28}$. A performance of 200,000 seconds and 0.07 times gravity allows one-way missions to the inner planets and asteroids to be completed in less than two weeks and to the outer planets and to Pluto in less than six months ${ }^{28}$. 
Table 2: Laser Propulsion Demonstration and Operational System Mass (kg) Note that indents are summed above

\begin{tabular}{|c|c|c|c|}
\hline Subsystem & $\begin{array}{c}\text { Ground Demo } \\
\text { System }\end{array}$ & $\begin{array}{l}\text { Flight } \\
\text { Demo }\end{array}$ & $\begin{array}{c}\text { Initial } \\
\text { Operational } \\
\text { System }\end{array}$ \\
\hline Bus/Structure & 43 & 34 & 54 \\
\hline Nozzle System & 18 & 18 & 29 \\
\hline Nozzle Coolant & 23 & 5 & 23 \\
\hline Nozzle Coolant Tank \& Feed System & 36 & 15 & 18 \\
\hline Thrust Structure & 7 & 5 & 5 \\
\hline Propellant (Pellets) Tank & 5 & 2 & 5 \\
\hline Propellant Feed System & 7 & 2 & 9 \\
\hline Lasers & 27 & 5 & 11 \\
\hline Power Supply & 50 & 18 & 36 \\
\hline Power Management System & 11 & 9 & 5 \\
\hline Instrumentation & 36 & 9 & 11 \\
\hline Instrumentation Data System & 43 & 9 & 9 \\
\hline Communication System & 18 & 27 & 39 \\
\hline Transmitter/Receiver & 18 & 11 & 11 \\
\hline Antennas & 0 & 16 & 27 \\
\hline Flight Control System & 0 & 13 & 28 \\
\hline Attitude Sensing & 0 & 3 & 3 \\
\hline Attitude Management & 0 & 5 & 7 \\
\hline Attitude Propellant (Cold gas) & 0 & 5 & 0 \\
\hline Attitude Management (Propellant) & 0 & 0 & 18 \\
\hline Ground Interfaces & 16 & 5 & 5 \\
\hline Launcher Interfaces - Physical & 0 & 5 & 5 \\
\hline Launcher Interfaces - Electrical/Electronic & 0 & 2 & 2 \\
\hline Thermal Management System & 18 & 14 & 20 \\
\hline Thermal Blankets & 0 & 7 & 9 \\
\hline Active Coolant & 0 & 5 & 7 \\
\hline Insulation & 18 & 2 & 5 \\
\hline Total Weight: & 358 & 197 & 317 \\
\hline
\end{tabular}

\section{Conclusion}

Laser-heated pellet pulse propulsion has the potential to compete with near-term deep space propulsion systems, but its ultimate potential is pulsed fusion propulsion. This makes LHPPP very attractive for development today. Systems for attitude control and orbital correction of geosynchronous satellites would provide the experience necessary to begin the development. If the solid pellets initially used became hollow, they could be filled with a mixture of deuterium and tritium which not only becomes an effective propellant, but also provides additional energy. The number of additional fusion reactions increases significantly with laser power, and also with frequency (i.e., larger photon momenta).. X-rays would be ideal, and could be generated using advanced free electron lasers. 
Momentum transfer to the pellet can be improved by using electron beams or ion beams. A magnetic pinch can be applied for additional compression. There are several other improvements that can be made. Cryogenic D-T fuel can be replaced with lithium-6 and deuterium if significant compression can be achieved. Igniting the pellet on the inside could provide significant magnetic fields to aid in plasma confinement. Adding a uranium shell would increase the inertial confinement and add neutrons. Lastly, antiproton pulses can be used for micro-fission. The fission fragments could be used to heat the fusion fuel; they would be confined by the self-generated magnetic fields at the annihilation sites. Only some of these improvements are required to develop viable fusion pulse propulsion, but it starts with a competitive laser-heated pellet pulse propulsion system.

\section{References}

${ }^{1}$ Mallove, E. and G. Matloff, The Starflight Handbook, John Wiley \& Sons, New York, 1989, pp.49-51.

${ }^{2}$ Forward, R.L., "Roundtrip Interstellar Travel Using Laser-Pushed Lightsails," Journal of Spacecraft and Rockets, 1984, pp. 187-195. 353.

${ }^{3}$ Kantrowitz, "Propulsion to Orbit by Ground-Based Laser," in Propulsion Techniques, edited by P.J. Turchi, 1998, pp. 351-

${ }^{4}$ Myrabo, L.N., and D. Ing, The Future of Flight, Simon \& Schuster, New York, 1985.

${ }^{5}$ Kammash, T, Fusion Reactor Physics, Ann Arbor Science, Ann Arbor, Michigan, 1982, pp. 263-295.

${ }^{6}$ WEISS, R.F, A.N. Pirri and N.H. Kemp, "Laser Propulsion" in Propulsion Techniques, edited by P.J. Turchi, 1998, pp. 355365. 1978.

${ }^{7}$ Project Daedalus Study Group, A., Bond, et al, Project Daedalus, Supplement, Journal of the British Interplanetary Society,

${ }^{8}$ Nicolson, I., The Road to the Stars, Westbridge Book, A Division of David \& Charles, Vancouver, BC, 1978.

${ }^{9}$ Chang Diaz, F.R., Developing VASIMIR, http://www.tamest.org/archive/2005/pdf/chang_diaz.pdf, January, 2005.

${ }^{10}$ Cassenti, B.N., "Engineering Challenges in Inertial Confinement Fusion Propulsion", Paper AIAA-2004-3533 presented at the 40th AIAA/ASME/SAE/ASEE Joint Propulsion Conference and Exhibit, Fort Lauderdale, Florida, July 11-14, 2004.

${ }^{11}$ Sutton, G.P., Rocket Propulsion Elements, 7th edition, John Wiley \& Sons, Inc., New York, p. 41, 2001.

${ }^{12}$ Miyamoto, K., Plasma Physics for Nuclear Fusion, Revised edition, The MIT Press, Cambridge, Section 16.7, 1987.

${ }^{13}$ McGuire, M.L., Borowski, S.K., Lee M. Mason, L.M., Gilland, J., "High Power MPD Nuclear Electric Propulsion (NEP)

${ }^{14}$ Kammash, T., Flippo, K., Umstadter, D., "Laser Accelerated Plasma Propulsion System (LAPPS)", JBIS, volume 58, No.11/12, p. 407, 2005.

${ }^{15}$ Nakano, M., Koizumi,H., Watanabe, M., and Arakawa, Y, "Laser Ignition Microthruster Experiments on KKS-1", Transactions of Space Technology Japan Volume 7, Issue ists26, pp. Pb_7-Pb_9 (2009).

${ }^{16}$ Hasegawa, A., Daido, H., Fujita, M., Mima, K., Murakami, M., Nakai, S., Nishihara, K., Terai, K., and Yamanaka, C., “Magnetically Insulated Inertially Confined Fusion - MICF," Nuclear Fusion, 28, pp. 369-387, (1988).

${ }^{17}$ Cassenti, B., "Lithium Hydride in Nuclear Propulsion", AIAA 2002-3920, 38th AIAA/ASME/SAE/ASEE Joint Propulsion Conference and Exhibit, Indianapolis, Indiana, 7-10 July, 2002.

${ }^{18}$ Davidson, R.C.,. Qin, Physics of Intense Charged Particle Beams, Imperial College Press, World Scientific, Singapore, 2001.

${ }^{19}$ Cassenti, B.N.. "Mass Production of Antimatter for High-Energy Propulsion", Journal of Propulsion and Power, Vol. 16, No. 1 (2000), pp. 119-124.

${ }^{20}$ Smith, G.A., et al, "Neutron yields and angular distributions produced in antiproton annihilation at rest in uranium”,Phys. Rev. C 45, 2332,1992

${ }^{21}$ Cassenti, B., and L. Coreano, "Antiproton Dispersion in Magnetically Insulated Inertial Confinement Fusion Propulsion", AIAA-2005-4135, 41st AIAA/ASME/SAE/ASEE Joint Propulsion Conference \& Exhibit, Tucson, Arizona, 10-13 July, 2005.

${ }^{22}$ Sterling, E., et al, Laser-Driven Mini-Thrusters, report Number AFRL-PR-ED-TP-2005-490, Air Force Research laboratory, Edwards AFB, CA,, 2005.

${ }^{23}$ Phipps, C., Laser Ablation Powered Mini-Thruster, High-Power Laser Ablation IV, Claude, R. Phipps, editor, Proceedings of the SPIE, Vol. 4760, pp 833-842, 2002.

${ }^{24}$ Sinko, J., Conical nozzles for pulsed laser propulsion, High-Power Laser Ablation VII, Claude, R. Phipps, editor, Proceedings of the SPIE, Vol. 7005, 70052Q, 2008.

${ }^{25} \mathrm{https}: / /$ lasers.llnl.gov/about/nif/

${ }^{26} \mathrm{http}: / /$ science.energy.gov/fes/

${ }^{27}$ Cassenti, B.N., "Trajectory Options for Manned Mars Missions", AIAA Journal of Spacecraft and Rockets, Vol. 42, No. 5, pp. 890-895, 2005.

${ }^{28}$ Cassenti, B.N., Kammash, T. Galbraith, D., "Antiproton Catalyzed Fusion Propulsion for Interplanetary Missions", AIAA Journal of Propulsion and Power, 13, No. 3, pp. 428-434, 1997. 